\title{
Rank two Vector Bundles on Elliptic Curves
}

\author{
CIARA DALY
}

\begin{abstract}
The aim of this paper is to give an overview of rank two vector bundles on an elliptic curve. It also aims to provide an outline of stability of vector bundles to serve as a motivation for the study of moduli spaces of vector bundles over elliptic curves. The first section outlines basic definitions and theorems. We will then study vector bundles on $\mathbb{P}^{1}$. From here, we go on to classify indecomposable rank two vector bundles over an elliptic curve. The final section introduces the notion of stability of vector bundles.
\end{abstract}

\section{INTRODUCTION}

Nowadays, vector bundles play an important role in many areas of mathematics such as algebraic geometry, algebraic topology and differential geometry, in the theory of partial differential equations.

The theory of vector bundles and the mathematical formalism developed over the years, for the study of vector bundle related concepts leads to the clarification or solution of many mathematical problems. Some of the vector bundle related concepts are generalisations of well-known classical notions. For instance, the notion of a section of a vector bundle over a space $X$ is a generalization of a vector valued function on $X$.

One of the important problems is the problem of classification of bundles. The problem of classification of vector bundles over an elliptic curve (i.e. a nonsingular projective curve of arithmetic genus one) has been completely solved by Atiyah in [1].

\section{Preliminaries}

For the purpose of this paper, $X$ will denote a complex manifold, unless otherwise specified. We will be working over $\mathbb{C}$, the field of complex numbers, throughout this paper. 
Definition 2.1. A complex vector bundle of rank $n$ is a holomorphic map $p: E \rightarrow X$ of complex manifolds which satisfy the following conditions:

(1) For any point $x \in X$, the preimage $E_{x}:=p^{-1}(x)$ (called a fibre) has a structure of an $\mathrm{n}$-dimensional $\mathbb{C}$-vector space.

(2) The mapping $\mathrm{p}$ is locally trivial, i.e. for any point $x \in X$, there exists an open neighbourhood $U_{i}$ containing $x$ and a biholomorphic map $\varphi_{i}: p^{-1}\left(U_{i}\right) \rightarrow U_{i} \times \mathbb{C}^{n}$ such that the diagram

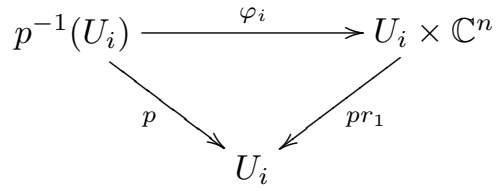

commutes.

Moreover, $\varphi_{i}$ takes the vector space $E_{x}$ isomorphically onto $\{x\} \times$ $\mathbb{C}^{n}$ for each $x \in U_{i} ; \varphi_{i}$ is called a trivialisation of $E$ over $U$. Note that for any pair of trivialisations $\varphi_{i}$ and $\varphi_{j}$, the map

$$
g_{i j}: U_{i} \cap V_{j} \rightarrow \mathrm{GL}(n, \mathbb{C})
$$

given by

$$
g_{i j}(x)=\varphi_{i} \circ\left(\left.\varphi_{j}\right|_{\{x\} \times \mathbb{C}^{n}}\right)^{-1}
$$

is holomorphic; the maps $g_{i j}$ are called transition functions for $E$ relative to the trivialisations $\varphi_{i}, \varphi_{j}$. The transition functions of $E$ necessarily satisfy the identities

$$
\begin{gathered}
g_{i j}(x) \cdot g_{j i}(x)=I \quad \text { for all } x \in U_{i} \cap U_{j} \\
g_{i j}(x) \cdot g_{j k}(x) \cdot g_{k i}(x)=I \quad \text { for all } x \in U_{i} \cap U_{j} \cap U_{k} .
\end{gathered}
$$

Conversely, given an open cover $\left\{U_{i}\right\}$ of $X$ and transition functions $g_{i j}: U_{i} \cap U_{j} \rightarrow \operatorname{GL}(n, \mathbb{C})$, for all $i, j$, then we can define a vector bundle, $E$ with transition functions $g_{i j}$ using the gluing construction as follows: We glue $U_{i} \times \mathbb{C}^{n}$ together by taking the union over all $i$ of $U_{i} \times \mathbb{C}^{n}$ to get $E:=\bigsqcup\left(U_{i} \times \mathbb{C}^{n}\right) / \sim$, where $(x, v) \sim\left(x, g_{i j}(x)(v)\right)$, for all $x \in U_{i} \cap U_{j}, v \in \mathbb{C}^{n}$.

A vector bundle of rank 1 is called a line bundle (See [7] Section 5 for more details). 
Example 2.2. The simplest example is known as the trivial vector bundle of rank $n$, i.e. $p r_{1}: X \times \mathbb{C}^{n} \rightarrow X$, where $p r_{1}$ denotes projection to the first factor.

The trivial line bundle on $X$, i.e. $X \times \mathbb{C} \rightarrow X$ will be denoted by $\mathcal{O}_{X}$, (or simply $\mathcal{O}$ if it is clear which $X$ we are referring to).

Example 2.3. The set $\mathcal{O}(-1) \subset \mathbb{P}^{n} \times \mathbb{C}^{n+1}$ that consists of all pairs $(\ell, z) \in \mathbb{P}^{n} \times \mathbb{C}^{n+1}$ with $z \in \ell$ forms in a natural way a line bundle over $\mathbb{P}^{n}$. To see this, consider the projection $p: \mathcal{O}(-1) \rightarrow \mathbb{P}^{n}$, where $p$ is the projection to the first factor. Let $\mathbb{P}^{n}=\bigcup_{i=0}^{n} U_{i}$ be the standard open covering. A canonical trivialisation of $\mathcal{O}(-1)$ over $U_{i}$ is given by $\varphi_{U_{i}}: p^{-1}\left(U_{i}\right) \cong U_{i} \times \mathbb{C},(\ell, z) \mapsto\left(\ell, z_{i}\right)$. The transition functions $g_{i j}(\ell): \mathbb{C} \rightarrow \mathbb{C}$ are given by $w \mapsto \frac{z_{i}}{z_{j}} \cdot w$, where $\ell=\left(z_{0}: \cdots: z_{n}\right)$.

Definition 2.4. Let $p: E \rightarrow X$ and $p^{\prime}: E^{\prime} \rightarrow X$ be two complex vector bundles on $X$. A holomorphic map $f: E \rightarrow E^{\prime}$ is called a morphism of vector bundles if the diagram

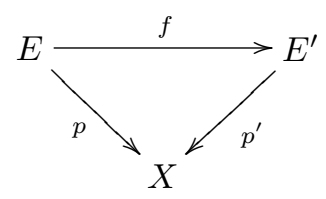

commutes and for each point $x \in X$ the map $\left.f\right|_{E_{x}}: E_{x} \rightarrow E_{x}^{\prime}$ is a homomorphism of vector spaces.

Let $E$ and $E^{\prime}$ be vector bundles over $X$ with rank $r$ and $r^{\prime}$, respectively and let $\left\{U_{i}\right\}$ be an open cover of $X$ such that $E$ and $E^{\prime}$ are trivial over $U_{i}$ for each $i$. A morphism $f: E \rightarrow E^{\prime}$ can be described locally by holomorphic functions, $f_{i}$, as follows. For each $i$, using trivialisations of $E$ and $E^{\prime}, f$ induces maps

$$
U_{i} \times \mathbb{C}^{r} \rightarrow U_{i} \times \mathbb{C}^{r^{\prime}}, \quad(x, v) \mapsto\left(x, f_{i}(x) v\right)
$$

where $f_{i}: U_{i} \rightarrow \operatorname{Mat}_{r^{\prime} \times r}(\mathbb{C})$. These holomorphic functions necessarily satisfy

$$
f_{i}(x) \cdot g_{i j}(x)=g_{i j}^{\prime}(x) \cdot f_{j}(x) \text { for all } x \in U_{i} \cap U_{j}
$$

where $g_{i j}$ and $g_{i j}^{\prime}$ are transition functions of $E$ and $E^{\prime}$, respectively. Note that a set of functions $\left\{f_{i}\right\}$ defines an isomorphism of vector bundles if an only if $f_{i}(x)$ are invertible matrices for all $i$ and $x$. 
Remark 2.5. It is important to note that this definition of a morphism of vector bundles does not make the category of vector bundles into an abelian category. For instance, if $f: E \rightarrow E^{\prime}$ is a morphism of vector bundles and the rank of $f$ is non-constant then $\operatorname{dim}\left(\operatorname{ker}\left(f_{x}\right)\right)$ jumps and so ker $f$ cannot form a vector bundle. The same would be true for coker $f$. On the contrary if $\operatorname{dim}\left(\operatorname{ker}\left(f_{x}\right)\right)$ is constant on $x$, then both ker $f$ and coker $f$ are vector bundles. This can be shown locally by a rank argument. It is necessary to use strict morphisms if an abelian category is required.

Definition 2.6. Let $E$ be a vector bundle on $X$ and let $\left\{U_{i}\right\}$ be an open cover of $X$. If the transition functions of $E$ are $g_{i j}$, then the dual bundle, $E^{*}$, of $E$ is given by transition functions

$$
h_{i j}(x):={ }^{t} g_{i j}(x)^{-1} \quad \forall x \in U_{i} \cap U_{j}
$$

Definition 2.7. A sequence of morphisms of vector spaces

$$
0 \longrightarrow A \stackrel{f}{\longrightarrow} B \stackrel{g}{\longrightarrow} C \longrightarrow 0
$$

is called a short exact sequence if $\operatorname{ker} g=\operatorname{im} f$, and if $f$ is injective and $g$ is surjective.

Definition 2.8. A sequence of morphisms of vector bundles over $X$

$$
0 \longrightarrow E^{\prime} \longrightarrow E \longrightarrow E^{\prime \prime} \longrightarrow 0
$$

is an exact sequence of vector bundles if

$$
0 \longrightarrow E_{x}^{\prime} \longrightarrow E_{x} \longrightarrow E_{x}^{\prime \prime} \longrightarrow 0
$$

is an exact sequence of vector spaces for all $x \in X$. The vector bundle $E^{\prime}$ is called a subbundle of $E$, and $E^{\prime \prime}$ is called a quotient bundle of $E$.

We also say that an exact sequence of vector bundles

$$
0 \longrightarrow E^{\prime} \longrightarrow E \longrightarrow E^{\prime \prime} \longrightarrow 0
$$

is an extension of $E^{\prime \prime}$ by $E^{\prime}$. In this case $E$ is called an extension of $E^{\prime \prime}$ by $E^{\prime}$.

Definition 2.9. Let $U$ be an open set in $X$. A holomorphic map $s: U \rightarrow E$ is called a holomorphic section of $E$ over $U$ if $p \circ s=i d_{U}$. Sections over $X$ are called global sections of $E$. Global sections can be added and multiplied with a scalar, so the space of global sections is in fact a vector space. It will be denoted by $H^{0}(X, E)$. 
Remark 2.10. Let $f: E^{\prime} \rightarrow E$ be a morphism of vector bundles. This induces a linear map of spaces of sections $H^{0}(f): H^{0}\left(E^{\prime}\right) \rightarrow H^{0}(E)$ by $H^{0}(f)\left(s^{\prime}\right):=f \circ s^{\prime}$.

2.1. Cohomology: Given a short exact sequence of vector bundles over $X$

$$
0 \longrightarrow E^{\prime} \stackrel{f}{\longrightarrow} E \stackrel{g}{\longrightarrow} E^{\prime \prime} \longrightarrow 0
$$

we can take global sections to get an exact sequence

$$
0 \longrightarrow H^{0}\left(X, E^{\prime}\right) \stackrel{H^{0}(f)}{\longrightarrow} H^{0}(X, E) \stackrel{H^{0}(g)}{\longrightarrow} H^{0}\left(X, E^{\prime \prime}\right)
$$

in which the last map $H^{0}(g): H^{0}(X, E) \rightarrow H^{0}\left(X, E^{\prime \prime}\right)$ is not in general surjective. For a counter example to $H^{0}(g)$ being surjective see [4] Chapter 8.

Since we get the exact sequence (1) above, we say that the global section functor is left exact. This global section sequence extends to a long cohomology exact sequence. For any vector bundle $E$ on $X$, the natural cohomology groups $H^{i}(X, E)$ (also denoted $H^{i}(E)$ if it is clear which $X$ we are referring to), for all $i>0$, can be defined satisfying the following property. Given a short exact sequence

$$
0 \longrightarrow E^{\prime} \longrightarrow E \longrightarrow E^{\prime \prime} \longrightarrow 0
$$

of vector bundles, there is an induced long exact sequence of cohomology groups

$$
0 \rightarrow H^{0}\left(X, E^{\prime}\right) \rightarrow H^{0}(X, E) \rightarrow H^{0}\left(X, E^{\prime \prime}\right) \rightarrow H^{1}\left(X, E^{\prime}\right) \rightarrow \cdots
$$

I will not define these cohomology groups in this paper, except to note that they exist and are very useful in computations. The dimension of $H^{i}(X, E)$ will be denoted $h^{i}(X, E)$. In the case of a curve $X$ the cohomology groups $H^{i}(X, E)$, vanish for all $i>1$, where 1 is the dimension of $X$, i.e. only the cohomology groups $H^{0}(X, E)$ and $H^{1}(X, E)$ are nonzero. (In fact $H^{i}(X, E)$ vanish for all $i>\operatorname{dim} X$ for a more general $X$ than just a curve though we do not need this fact in this paper. See [4] Chapter 8 for more details).

2.2. Ext groups: If $E$ and $E^{\prime}$ are vector bundles over $X$, we denote by $\operatorname{Hom}_{X}\left(E, E^{\prime}\right)$ (or $\operatorname{Hom}\left(E, E^{\prime}\right)$ if it is clear which $X$ we are referring to) the vector space of vector bundle morphisms. For a fixed $E, \operatorname{Hom}(E, \cdot)$ is a left exact covariant functor from the category of vector bundles to the category of vector spaces, i.e. given a short exact sequence of vector bundles

$$
0 \longrightarrow F^{\prime} \longrightarrow F \longrightarrow F^{\prime \prime} \longrightarrow 0
$$


we get another exact sequence in which the last map is not surjective in general

$$
0 \longrightarrow \operatorname{Hom}\left(E, F^{\prime}\right) \longrightarrow \operatorname{Hom}(E, F) \stackrel{g}{\longrightarrow} \operatorname{Hom}\left(E, F^{\prime \prime}\right)
$$

And so in a similar fashion to the way we defined cohomology groups $H^{i}(E)$, we can define what are called the Ext groups, which allow us to extend our short exact sequence (2) to a long exact sequence as follows:

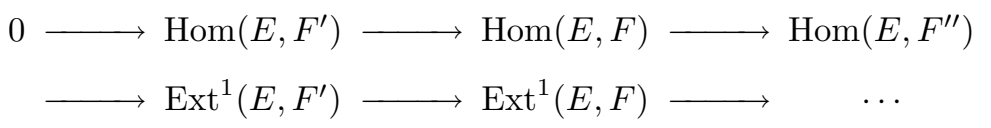

We say that $\operatorname{Ext}^{i}(E, \cdot)$ are the right derived functors of $\operatorname{Hom}(E, \cdot)$. So in particular we have $\operatorname{Ext}^{0}(E, \cdot)=\operatorname{Hom}(E, \cdot)$. We have the following proposition to see the relationship between the cohomology groups $H^{i}$ and the Ext groups (for a full proof of this proposition see [5] Proposition 6.3).

Proposition 2.11. For any vector bundle $E$ on a complex manifold $X$ we have:

$\operatorname{Ext}^{i}\left(\mathcal{O}_{X}, E\right) \cong H^{i}(E)$ for all $i \geq 0$.

Similarly we have:

$\operatorname{Ext}^{i}\left(E, \mathcal{O}_{X}\right) \cong H^{i}\left(E^{*}\right)$ for all $i \geq 0$.

Proof. Here we will just give a proof of the first statement, where $i=0$. Let $f \in \operatorname{Hom}(\mathcal{O}, E)$, a fibre-wise holomorphic morphism such that the following diagram commutes

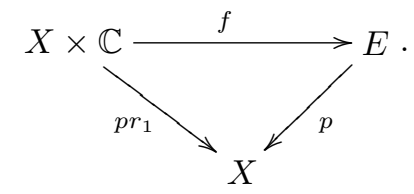

Let $s: X \rightarrow E$ be a holomorphic section of $E$, i.e. $p \circ s=\mathrm{id}_{x}$. Define two linear maps

$$
\alpha: \operatorname{Hom}(\mathcal{O}, E) \rightarrow H^{0}(E)
$$

and

$$
\beta: H^{0}(E) \rightarrow \operatorname{Hom}(\mathcal{O}, E)
$$

as follows: Define $\alpha(f)(x):=f(x, 1)$ and $\beta(s)(x, \lambda):=\lambda \cdot s(x)$.

We see that $\beta(\alpha(f))=f$ as follows: 
$\beta(\alpha(f))(x, \lambda)=\lambda \cdot(\alpha(f)(x))=\lambda \cdot f(x, 1)=f(x, \lambda)$, where the last equality uses the fact that $f$ is linear on fibres.

Similarly we obtain $\alpha(\beta(s))(x)=\beta(s)(x, 1)=s(x)$, so $\alpha(\beta(s))=$ $s$. Hence $\alpha$ and $\beta$ are inverses of one another and we get

$$
\operatorname{Hom}(\mathcal{O}, E) \cong H^{0}(E)
$$

Remark 2.12. Given a vector bundle $E$, and given that $H^{0}(E) \neq 0$, then from Proposition 2.11 we know that $\operatorname{Hom}(\mathcal{O}, E) \neq 0$. So we have $f: \mathcal{O} \rightarrow E$. We can say that $\mathcal{O} \subset E$, though here ' $\subset$ ' does not denote a subbundle, but rather a 'subsheaf'. Vector bundles can also be described as sheaves (in particular locally free sheaves) though we have not built up this language in this paper. It suffices to know that if $\mathcal{O} \subset E$ as a subsheaf then we can extend this to get a line subbundle $M \subset E$ on $X$, a smooth curve (See [9] Chapter 10 for more details). This will be useful in proofs later on.

The notion of a degree of a line bundle on a curve was introduced in [7] (Section 5). We can extend this definition to vector bundles of arbitrary rank. To do so we must first define the determinant line bundle.

Definition 2.13. Given a vector bundle $E$ of rank $r$, it's determinant line bundle is defined to be the $r$-th exterior power of $E$, denoted:

$$
\operatorname{det} E:=\wedge^{r} E \text {. }
$$

where the fibres of $X$ for any $x \in X$ are canonically isomorphic to $\wedge^{r} E_{x}$.

For those of you who are unfamiliar with exterior power, we can reformulate the definition as follows: Given an open cover $\left\{U_{i}\right\}$ of $X$ and a vector bundle $E$ over $X$ with transition functions $g_{i j}$, the determinant line bundle of $E$ is given by transition functions $h_{i j}$ where

$$
h_{i j}(x):=\operatorname{det} g_{i j}(x) \in \mathrm{GL}(1, \mathbb{C}), \quad \text { for all } x \in U_{i} \cap U_{j}
$$

This now allows us to define the degree of a vector bundle as follows.

Definition 2.14. The degree $\operatorname{deg} E \in \mathbb{Z}$ of a vector bundle is the degree of its determinant line bundle $\operatorname{det} E$. 
Remark 2.15. Recall from [7] that the degree of a line bundle is the degree of it's associated divisor and every line bundle can be written as $\mathcal{O}(D)$, with $D$ a divisor on $X$.

If $E$ lies in an exact sequence of vector bundles on $X$ as follows:

$$
0 \rightarrow E^{\prime} \rightarrow E \rightarrow E^{\prime \prime} \rightarrow 0
$$

then there is an isomorphism

$$
\operatorname{det} E^{\prime} \otimes \operatorname{det} E^{\prime \prime} \cong \operatorname{det} E .
$$

Since $\operatorname{det} E^{\prime}$ and $\operatorname{det} E^{\prime \prime}$ are line bundles we get $\operatorname{deg} E=\operatorname{deg} E^{\prime}+$ $\operatorname{deg} E^{\prime \prime}$ (as in general $\operatorname{deg}\left(L \otimes L^{\prime}\right)=\operatorname{deg} L+\operatorname{deg} L^{\prime}$, where $L$ and $L^{\prime}$ line bundles). In other words, degree is additive on exact sequences. As a special case, if a vector bundle $E=L_{1} \oplus L_{2}$ is the direct sum of two line bundles $L_{1}$ and $L_{2}$, then we have

$$
0 \rightarrow L_{1} \rightarrow E \rightarrow L_{2} \rightarrow 0
$$

with $\operatorname{deg} E=\operatorname{deg} L_{1}+\operatorname{deg} L_{2}$.

Remark 2.16. Let $E$ be a vector bundle of rank $r$ over a complex manifold $X$. If we tensor $E$ with a line bundle $L$, then $\operatorname{deg}(E \otimes L)=$ $\operatorname{deg} E+r \operatorname{deg} L$. In particular for $E$ of rank 2 we have $\operatorname{deg}(E \otimes L)=$ $\operatorname{deg} E+2 \operatorname{deg} L$. To see this let's look at an example:

Let $E=L_{1} \oplus L_{2}$ for line bundles $L_{1}$ and $L_{2}$. Tensor this with another line bundle $L$ to get

$$
E \otimes L=\left(L_{1} \oplus L_{2}\right) \otimes L=\left(L_{1} \otimes L\right) \oplus\left(L_{2} \otimes L\right)
$$

Now

$$
\begin{aligned}
\operatorname{deg}(E \otimes L) & =\operatorname{deg}\left(L_{1} \otimes L\right)+\operatorname{deg}\left(L_{2} \otimes L\right) \\
& =\operatorname{deg} L_{1}+\operatorname{deg} L+\operatorname{deg} L_{2}+\operatorname{deg} L \\
& =\operatorname{deg} L_{1}+\operatorname{deg} L_{2}+2 \operatorname{deg} L \\
& =\operatorname{deg} E+2 \operatorname{deg} L
\end{aligned}
$$

In this way, when considering vector bundles of rank 2 , it is enough to consider vector bundles of degree -1 or 0 (or indeed, any even and odd degree), then by tensoring with a line bundle of appropriate degree we get all other degrees. We call this the "tensor product trick".

Definition 2.17. An exact sequence of vector bundles

$$
0 \rightarrow E^{\prime} \rightarrow E \rightarrow E^{\prime \prime} \rightarrow 0
$$


splits if and only if there exists a homomorphism $f: E^{\prime \prime} \rightarrow E$ for which the composition $E^{\prime \prime} \stackrel{f}{\longrightarrow} E \longrightarrow E^{\prime \prime}$ is an isomorphism.

In this case, the map $f$ is called a splitting of the sequence.

Now consider, on any curve, a short exact sequence of vector bundles

$$
\mathbb{E}: \quad 0 \longrightarrow M \stackrel{\alpha}{\longrightarrow} E \stackrel{\beta}{\longrightarrow} L \longrightarrow 0 .
$$

By applying $\operatorname{Hom}(L,-)$ to this sequence we get the following morphism:

$$
\operatorname{Hom}(L, L) \stackrel{\delta}{\longrightarrow} \operatorname{Ext}^{1}(L, M)
$$

Definition 2.18. The image under the coboundary map $\delta$ of $\operatorname{id}_{L} \in$ $\operatorname{Hom}(L, L)$, which we will denote by

$$
\delta\left(\operatorname{id}_{L}\right) \in \operatorname{Ext}^{1}(L, M) \cong H^{1}\left(L^{*} \otimes M\right)
$$

is called the extension class of $\mathbb{E}$.

By exactness of

$$
\operatorname{Hom}(L, E) \stackrel{\rho}{\longrightarrow} \operatorname{Hom}(L, L) \stackrel{\delta}{\longrightarrow} \operatorname{Ext}^{1}(L, M),
$$

if $\delta\left(\operatorname{id}_{L}\right)=0$, then there exists a homomorphism $f: L \rightarrow E$ for which the $\operatorname{id}_{L}=\beta \circ f: L \rightarrow L$, i.e. the sequence $\mathbb{E}$ splits. Moreover, if $\mathbb{E}$ splits, there exists $f: L \rightarrow E$ such that $\operatorname{id}_{L}=\beta \circ f$. Because the composition, $\rho \circ \delta$ is zero, we have $\delta\left(\operatorname{id}_{L}\right)=0$. Hence we have the following proposition:

Proposition 2.19. The sequence $\mathbb{E}$, i.e.

$$
0 \rightarrow M \rightarrow E \rightarrow L \rightarrow 0 .
$$

splits if and only if $\operatorname{Ext}^{1}(L, M)=0$. In particular, if $\operatorname{Ext}^{1}(L, M) \cong$ $H^{1}\left(L^{*} \otimes M\right)=0$, then every exact sequence $\mathbb{E}$ splits.

Remark 2.20. For each $\alpha \in \operatorname{Ext}^{1}\left(E^{\prime \prime}, E^{\prime}\right)$ there exists an extension

$$
0 \rightarrow E^{\prime} \rightarrow E_{\alpha} \rightarrow E^{\prime \prime} \rightarrow 0
$$

with a vector bundle, $E_{\alpha}$, in such a way that $\alpha$ is the extension class of (3). Moreoever, $E_{\alpha} \cong E_{\beta}$ if and only if there exists $\lambda \in \mathbb{C}^{*}$ such that $\alpha=\lambda \beta$. (See [10] Section 3.4 for more details) 
2.3. Riemann-Roch Formula for Curves. We have a very useful tool, called the Riemann-Roch formula, which tells us a lot about the cohomology groups of a vector bundle $E, H^{0}(E)$ and $H^{1}(E)$, once we know the rank and degree of $E$. The Riemann-Roch formula is as follows: If $E$ is a vector bundle of rank $r$ on a curve of genus $g$, then:

$$
h^{0}(E)-h^{1}(E)=\operatorname{deg} E-r(g-1) .
$$

In addition to the Riemann-Roch formula, one of the other major tools we have in dealing with cohomology is Serre duality. The following proposition outlines Serre duality, though will not be proved as the proof is too involved for this paper.

Proposition 2.21. (Serre duality) Let $X$ be a smooth projective curve. Let $E$ be a vector bundle on $X$. Let $K_{X}$ be a canonical line bundle on $X$. Then there are canonical isomorphisms

$$
H^{0}(X, E) \cong H^{1}\left(X, K_{X} \otimes E^{*}\right)^{*} .
$$

and

$$
H^{1}(X, E) \cong H^{0}\left(X, K_{X} \otimes E^{*}\right)^{*} .
$$

In particular it follows that $H^{0}(X, E)$ and $H^{1}\left(X, K_{X} \otimes E^{*}\right)$ have the same dimension.

While I have not defined $K_{X}$, the canonical line bundle, for the purpose of this paper it will suffice to know what $K_{X}$ is in the case of a curve. This is outlined below:

$$
\begin{gathered}
X=\mathbb{P}^{1}: \quad K_{X}=\mathcal{O}_{\mathbb{P}^{1}}(-2), \quad \operatorname{deg}\left(K_{X}\right)=-2 \\
X=\text { elliptic curve }: \quad K_{X}=\mathcal{O}_{X}, \quad \operatorname{deg}\left(K_{X}\right)=0 \\
X=\text { curve of genus } g \geq 2: \quad \operatorname{deg}\left(K_{X}\right)=2 g-2 .
\end{gathered}
$$

Refer to [5] Section III.7 for more details on Serre duality.

Lemma 2.22. Let $L$ be a line bundle on a curve, $C$, of genus $g$. Then we have the following:
(a) $H^{0}(L)=0$ if $\operatorname{deg} L<0$.
(b) $H^{1}(L)=0$ if $\operatorname{deg} L>2 g-2$.
(c) $L \cong \mathcal{O}$ if $\operatorname{deg} L=0$ and $s \in H^{0}(L), s \neq 0$.

The proof of (a) and (c) of the lemma above uses the correspondence between line bundles and divisors (see again [7]) and the fact that the divisor defined by a nonzero holomorphic section of a line bundle is always positive. The proof of (b) follows from Serre duality and part (a). 
Lemma 2.23. If $E$ is a vector bundle on a curve $C$ of genus $g$, then the degree of its subbundles $F \subset E$ is bounded above.

Proof. ([9], Corollary 10.9) Since the global sections functor is left exact, we get

$$
H^{0}(F) \subset H^{0}(E)
$$

This implies that $h^{0}(F) \leq h^{0}(E)$. Now by Riemann-Roch we know

$$
h^{0}(F)-h^{1}(F)=\operatorname{deg}(F)+\operatorname{rk}(F) \cdot(1-g) .
$$

From this we get

$$
\operatorname{deg}(F)+\operatorname{rk}(F) \cdot(1-g)+h^{1}(F) \leq h^{0}(E)
$$

and by rearranging we have

$$
\operatorname{deg}(F) \leq h^{0}(E)-\operatorname{rk}(F) \cdot(1-g)-h^{1}(F)
$$

Now if $g=1$, we see that $\operatorname{deg}(F) \leq h^{0}(E)-h^{1}(F)$ and since $h^{1}(F) \geq 0$, we get $\operatorname{deg}(F) \leq h^{0}(E)$.

If $g=0$, then $\operatorname{deg}(F) \leq h^{0}(E)-\operatorname{rk}(F)-h^{1}(F)$ and since $\operatorname{rk}(F) \geq 0$ and $h^{1}(F) \geq 0$, we see that $\operatorname{deg}(F) \leq h^{0}(E)$.

If $g \geq 2, \operatorname{deg}(F) \leq h^{0}(E)+\operatorname{rk}(F) \cdot(g-1)-h^{1}(F) \leq h^{0}(E)+\operatorname{rk}(E)$. $(g-1)-h^{1}(F)($ since $\operatorname{rk}(F) \leq \operatorname{rk}(E))$. Again, since $h^{1}(F) \geq 0$, we get $\operatorname{deg}(F) \leq h^{0}(E)+\operatorname{rk}(E) \cdot(g-1)$. Hence we see that in any case the degree of $F \subset E$ is bounded above.

\section{Vector Bundles on $\mathbb{P}^{1}$}

Before we move on to vector bundles on an elliptic curve (i.e. a curve of genus one), it makes sense to look at vector bundles on a curve of genus zero $\left(\mathbb{P}^{1}\right)$. Let us now restate Lemma 2.22 in the case of $\mathbb{P}^{1}$, where genus $g=0$, to see how the cohomology of line bundles on $\mathbb{P}^{1}$ is particularly simple.

Lemma 3.1. Let $L$ be a line bundle on $\mathbb{P}^{1}$. Then we have the following:

(a) $H^{0}(L)=0$ if $\operatorname{deg} L \leq-1$.

(b) $H^{1}(L)=0$ if $\operatorname{deg} L \geq-1$.

By Riemann-Roch we also have,

$$
h^{0}(L)-h^{1}(L)=\operatorname{deg} L+1 .
$$

Remark 3.2. We have seen from [7] that for $L$ a line bundle, $L^{*}$ is the inverse of the line bundle $L$ in the Picard group. We have also seen that deg : Pic $X \rightarrow \mathbb{Z}$ is a homomorphism (where Pic $X$ denotes the set of line bundles over $X$ ) and so we get $\operatorname{deg} L^{*}=-\operatorname{deg} L$. 
Lemma 3.3. The homomorphism $\operatorname{deg}: \operatorname{Pic} \mathbb{P}^{1} \rightarrow \mathbb{Z}$ is an isomorphism.

Proof. See [4] Lemma 6.2.11.

We have a classification for all vector bundles on $\mathbb{P}^{1}$ as follows:

Lemma 3.4. Every rank 2 vector bundle on $\mathbb{P}^{1}$ is isomorphic to a direct sum of two line bundles

Proof. ([9], Lemma 10.30) Let $E$ be a rank 2 vector bundle on $\mathbb{P}^{1}$. Tensoring with a line bundle if necessary, it is enough to assume that $\operatorname{deg} E=0$ or -1 . First, by the Riemann-Roch formula we note that $H^{0}(E) \neq 0$, and so from Remark 2.12 above we get a line bundle $M \subset E$, and $M \cong \mathcal{O}(D)$ for some positive divisor $D \geq 0$. In particular, $\operatorname{deg} M \geq 0$, and denoting the quotient by $L:=E / M$, we have an exact sequence

$$
0 \rightarrow M \rightarrow E \rightarrow L \rightarrow 0 .
$$

Now $\operatorname{deg} E=\operatorname{deg} L+\operatorname{deg} M$, hence $\operatorname{deg}\left(L^{*} \otimes M\right)=\operatorname{deg} M-\operatorname{deg} L=$ $-\operatorname{deg} E+2 \operatorname{deg} M \geq-\operatorname{deg} E \geq 0$. From Lemma 3.1 (b), we get $H^{1}\left(L^{*} \otimes M\right)=0$. By Proposition 2.19, therefore, the sequence splits.

Grothendieck's Theorem 3.5. Every vector bundle on $\mathbb{P}^{1}$ is isomorphic to a direct sum of line bundles.

Proof. ([9] Theorem 10.31) Let $E$ be a vector bundle of rank $r$ on $\mathbb{P}^{1}$. Proof is by induction on the rank $r \geq 2$ of $E$, starting with the previous lemma. Serre's Theorem ([5] II.5.17) tells us that there exists a line subbundle in $E$. Now let $M \subset E$ be the line subbundle whose degree, $m=\operatorname{deg} M$, is maximal among line subbundles of $E$ (Lemma 2.23). Let $F:=E / M$ be a vector bundle of rank $r-1$.

Claim: Every line subbundle $L \subset F$ has $\operatorname{deg} L \leq m$.

Now we have a short exact sequence as follows:

$$
0 \rightarrow M \rightarrow E \rightarrow F \rightarrow 0
$$

By considering the preimage $\tilde{L} \subset E$ of $L$ under the quotient morphism $E \rightarrow F$ we get a diagram as follows:

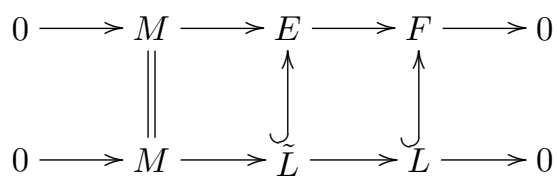


Clearly $\tilde{L}$ is a rank 2 vector bundle, and we see $\operatorname{deg} \tilde{L}=m+\operatorname{deg} L$. By Lemma 3.4, we know $\tilde{L} \cong L_{1} \oplus L_{2}$ for some line bundles $L_{1}$ and $L_{2}$. Now $\operatorname{deg}(\tilde{L})=\operatorname{deg}\left(L_{1}\right)+\operatorname{deg}\left(L_{2}\right)$ so one of $L_{1}$ or $L_{2}$ must have deg at least $\operatorname{deg}(\tilde{L}) / 2$. Let $N$ denote that line subbundle, of degree at least $\operatorname{deg} \tilde{L} / 2$. Because $N$ is a subbundle of $E$, as well as our choice of $M$ we get $m \geq \operatorname{deg} N \geq(\operatorname{deg} \tilde{L}) / 2=\frac{m+\operatorname{deg} L}{2}$ and the claim follows easily from this.

By the inductive hypothesis, we know the quotient bundle $F$ is isomorphic to a direct sum $F=L_{1} \oplus \cdots \oplus L_{r-1}$ of line bundles and the claim gives us $\operatorname{deg} L_{i} \leq m$. Since $H^{1}\left(L_{i}^{*} \otimes M\right)=0$ for each $i$. It follows that the exact sequence

$$
0 \rightarrow M \rightarrow E \rightarrow \bigoplus_{i=1}^{r-1} L_{1} \rightarrow 0
$$

splits.

Definition 3.6. A vector bundle, $E$, is called decomposable if it is isomorphic to the direct sum $E_{1} \oplus E_{2}$ of two nonzero vector bundles; otherwise, $E$ is called indecomposable.

By definition of decomposability, every vector bundle is the direct sum of indecomposable ones. Therefore, it suffices to know the indecomposable vector bundles on a curve in order to know them all. We have seen that all vector bundles on rational curves are the direct sum of line bundles. As well as the notion of an indecomposable vector bundle, we also have the notion of a simple vector bundle.

Definition 3.7. A vector bundle $E$ is simple if its only endomorphisms are scalars, End $E=\mathbb{C}$. Every line bundle is simple.

A simple vector bundle is necessarily indecomposable. To see this let us start with a decomposable vector bundle $E \oplus F$. Consider $f: E \oplus F \rightarrow E \oplus F$, where $f=\operatorname{id}_{E} \oplus 0_{F}$ where $\operatorname{id}_{E}$ is the identity map on $\mathrm{E}$ and $0_{F}$ is the zero map on $F$. Clearly then $\operatorname{End}(E \oplus F) \neq \mathbb{C}$, i.e., $F$ is not simple.

Note that the converse is not true, i.e. an indecomposable vector bundle is not necessarily simple (This can be seen by a counterexample, Example 4.4 below). 


\section{Classification of all Indecomposable Rank two Vector Bundles on an Elliptic Curve $C$}

We are now ready to look at the case of a nonsingular curve of arithmetic genus one (i.e. an elliptic curve). Atiyah's paper of 1957 ([1]) provided us with an answer to this case. We have already seen in Lemma 3.3 that there is exactly one line bundle on $\mathbb{P}^{1}$ for every degree. In particular $\operatorname{Pic}^{0}\left(\mathbb{P}^{1}\right)=\{\mathcal{O}\}$, where $\operatorname{Pic}^{0}\left(\mathbb{P}^{1}\right)$ denotes the set of line bundles of degree 0 on $\mathbb{P}^{1}$. However it turns out ([7] Theorem 20) on an elliptic curve, $C$, that $\operatorname{Pic}^{0}(C)$ is in bijection to $C$ and so on elliptic curves there are more vector bundles in the sense that nontrivial extensions appear. For the purpose of this paper we will be concentrating on rank 2 vector bundles on an elliptic curve. In this section we will give a classification of all indecomposable rank 2 vector bundles on the elliptic curve $C$.

First let me return to the Riemann-Roch formula for a vector bundle $E$, this time looking at a curve of genus 1, i.e.

$$
h^{0}(E)-h^{1}(E)=\operatorname{deg} E
$$

Note that every line bundle, $L$, on $C$ satisfies:

$$
h^{0}(L)-h^{1}(L)=\operatorname{deg} L
$$

The next lemma follows from the above equation and Lemma 2.22:

Lemma 4.1. Let $L$ be a line bundle on an elliptic curve. Then we have the following:

(a) $H^{0}(L)=0$ if $\operatorname{deg} L<0$.

(b) $H^{1}(L)=0$ if $\operatorname{deg} L>0$.

(c) If $\operatorname{deg} L=0$ and $L \neq \mathcal{O}$, then $H^{0}(L)=H^{1}(L)=0$.

Lemma 4.2. If $E$ is an indecomposable vector bundle of rank 2 on a smooth projective curve, $X$, then every line subbundle $L \subset E$ satisfies

$$
2 \operatorname{deg} L \leq \operatorname{deg} E+2 g-2
$$

Proof. Let $M$ be the quotient line bundle $E / L$. This gives us the following short exact sequence:

$$
0 \rightarrow L \rightarrow E \rightarrow M \rightarrow 0
$$

which corresponds to an element in $\operatorname{Ext}^{1}(M, L) \cong H^{1}\left(M^{*} \otimes L\right)$. Now since $E$ is indecomposable, this sequence cannot split and hence $H^{1}\left(M^{*} \otimes L\right) \neq 0$ by Proposition 2.19. By Serre duality, this implies that $0 \neq H^{0}\left(\left(M^{*} \otimes L\right)^{*} \otimes K_{X}\right)^{*}=H^{0}\left(M \otimes L^{*} \otimes K_{X}\right)^{*}$. This in turn 
implies that $\operatorname{deg}\left(M \otimes L^{*} \otimes K_{X}\right)=\operatorname{deg} M-\operatorname{deg} L+2 g-2 \geq 0$ from Lemma 2.22 (a). Now from the short exact sequence above we know that $\operatorname{deg} E=\operatorname{deg} M+\operatorname{deg} L$, i.e. $\operatorname{deg} M=\operatorname{deg} E-\operatorname{deg} L$. Hence we get

$$
\operatorname{deg} E-\operatorname{deg} L-\operatorname{deg} L+2 g-2 \geq 0
$$

From this, we get the inequality in the lemma.

Let $\mathcal{E}(r, d)$ denote the set of isomorphism classes of indecomposable vector bundles of rank $r$ and degree $d$ over $X$, an elliptic curve.

Theorem 4.3. (a) There exists a vector bundle $E_{r} \in \mathcal{E}(r, 0)$, unique up to isomorphism, with $H^{0}\left(E_{r}\right) \neq 0$. Moreover, we have an exact sequence:

$$
0 \rightarrow \mathcal{O}_{X} \rightarrow E_{r} \rightarrow E_{r-1} \rightarrow 0
$$

(b) Let $E \in \mathcal{E}(r, 0)$, then $E \cong E_{r} \otimes L$, where $L$ is a line bundle of degree zero, unique up to isomorphism.

Proof. See [1] Theorem 5.

Example 4.4. The bundles $E_{r}$ of Theorem 4.3 are sometimes called the Atiyah bundles. For $r \geq 2$, they are examples of indecomposable vector bundles which are not simple. Let us prove now that $E_{2}$ is not simple.

We know $E_{2}$ sits in an exact sequence as follows:

$$
0 \longrightarrow \mathcal{O}_{X} \stackrel{f}{\longrightarrow} E_{2} \longrightarrow \mathcal{O}_{X} \longrightarrow 0
$$

Applying $\operatorname{Hom}\left(-, E_{2}\right)$ to this sequence, we get

$$
0 \longrightarrow \operatorname{Hom}\left(\mathcal{O}, E_{2}\right) \longrightarrow \operatorname{Hom}\left(E_{2}, E_{2}\right) \stackrel{\beta}{\longrightarrow} \operatorname{Hom}\left(\mathcal{O}, E_{2}\right)
$$

Now $\operatorname{Hom}\left(\mathcal{O}, E_{2}\right) \cong H^{0}\left(E_{2}\right)$ from Proposition 2.11. From our assumption on $E_{2}$, we know $H^{0}\left(E_{2}\right) \neq 0$, i.e. $h^{0}\left(E_{2}\right) \geq 1$. Now let $\operatorname{id}_{E_{2}}$ denote $\mathrm{id} \in \operatorname{Hom}\left(E_{2}, E_{2}\right)$. We know, under the morphism $\beta$, that $\operatorname{id}_{E_{2}} \mapsto f \in \operatorname{Hom}\left(\mathcal{O}, E_{2}\right) \neq 0$, i.e. $\beta\left(\operatorname{id}_{E_{2}}\right)=f \neq 0$. This implies $\beta \neq 0$. So we get the following short exact sequence:

$$
0 \rightarrow \operatorname{Hom}\left(\mathcal{O}, E_{2}\right) \rightarrow \operatorname{Hom}\left(E_{2}, E_{2}\right) \rightarrow \operatorname{im}(\beta) \rightarrow 0
$$

Since $\beta \neq 0$, we know that $\operatorname{dim}(\operatorname{im}(\beta)) \geq 1$. Now since $\operatorname{dim}$ is additive on exact sequences, $\operatorname{dim}\left(\operatorname{Hom}\left(E_{2}, E_{2}\right)\right)=\operatorname{dim}\left(\operatorname{Hom}\left(\mathcal{O}, E_{2}\right)\right)+$ $\operatorname{dim}(\operatorname{im}(\beta)) \geq 2$. Hence by the definition of a simple vector bundle (Definition 3.7), we know that $E_{2}$ is not simple. 
Let us now classify all indecomposable rank 2 vector bundles on an elliptic curve. We first consider the case of even degree.

Proposition 4.5. On a curve, $C$, of genus 1 every indecomposable rank 2 vector bundle, $E$, of even degree is an extension of the form

$$
0 \longrightarrow M \longrightarrow E \longrightarrow M \longrightarrow 0
$$

for some line bundle $M$ on $E$

Proof. ([9] Proposition 10.48) Using the tensor product trick, it is enough to consider the case where $\operatorname{deg} E=2 k$. If $M_{1} \in \operatorname{Pic}^{k}(C)$, i.e. $M_{1}$ is a line bundle of degree $k$, then $E \otimes M_{1}$ is of degree 0 . In other words, $E \otimes M_{1} \in \mathcal{E}(r, 0)$. By Theorem 4.3 , we know that there exists $M_{2} \in \operatorname{Pic}^{0}(C)$ such that $E \otimes M_{1} \cong E_{2} \otimes M_{2}$, where $E_{2}$ is the so-called Atiyah bundle from Theorem 4.3. Then $E_{2}$ sits in a non-split exact sequence as follows

$$
0 \rightarrow \mathcal{O}_{C} \rightarrow E_{2} \rightarrow \mathcal{O}_{C} \rightarrow 0 .
$$

If $M:=M_{2} \otimes M_{1}^{*}$, we obtain $E \cong E_{2} \otimes M$ and tensoring this sequence by $M$, gives a short exact sequence

$$
0 \rightarrow M \rightarrow E \rightarrow M \rightarrow 0 \text {. }
$$

The following proposition contains the odd degree case.

Proposition 4.6. On a curve, $C$, of genus 1 , given a line bundle $L$ of odd degree, there exists, up to isomorphism, a unique indecomposable rank 2 vector bundle $E$ with $\operatorname{det} E \cong L$.

We refer to [9] Proposition 10.47 for the proof.

Theorem 4.7. For each integer $n$, there is a one-to-one correspondence between the set of isomorphism classes of indecomposable vector bundles of rank 2 and degree $n$ on the elliptic curve $C$, and the set of points on $C$.

Sketch of correspondence We will denote by $\operatorname{Pic}^{n}(C)$, the set of degree $n$ line bundles on $C$. Recall from [7], Theorem 20, that there is an isomorphism of manifolds $C \cong \operatorname{Pic}^{0}(C)$ and in fact ([7], Theorem 21) there is an isomorphism $C \cong \operatorname{Pic}^{n}(C)$ for all $n \in \mathbb{Z}$.

Now let $E$ be an indecomposable rank 2 vector bundle of degree $n$ on $C$. If $n$ is odd, from Proposition 4.6, we know that there is a unique indecomposable rank 2 vector bundle $E$ of degree $n$, with $\operatorname{det} E \cong L$. Hence use $\operatorname{Pic}^{n}(C) \cong C$ to obtain the result. 
If $n$ is even from Theorem 4.3 we know there exists, $L$, a line bundle of degree zero, unique up to isomorphism such that $E \otimes L$ is isomorphic to the unique nontrivial extenstion of $\mathcal{O}_{C}$ by $\mathcal{O}_{C}$. Since $\operatorname{Pic}^{0}(C) \cong C$ again we obtain the result.

\section{StabiLity}

The notion of stability comes from the theory of moduli spaces. The variety, $\operatorname{Pic}^{0}(C)$ with the Poincaré bundle, of degree 0 line bundles on an elliptic curve (See [7] Section 6) is an example of a moduli space. Loosely described a moduli space is an algebraic variety which parametrises the set of equivalence classes of some objects. For example we could consider the moduli space of rank two vector bundles on an elliptic curve, $C$. It turns out that the set of isomorphism classes of vector bundles of rank 2 and degree $d$ on an elliptic curve is unbounded (briefly, this means that we can find families of arbitrarily high dimension which gives us vector bundles of rank 2 and degree $d$. See [6] Chapter 1), which poses a problem when constructing the corresponding moduli space. To overcome this problem we restrict our study of vector bundles. One form of restriction is to study 'stable' vector bundles. Using stable bundles, one to construct the moduli space of (stable) vector bundles of rank 2 and degree $d$ on $C$.

We will begin by giving a more explicit definition of a subbundle and quotient vector bundle.

Definition 5.1. Let $F$ and $E$ be vector bundles of rank $r$ and $n$ respectively, with $r \leq n$ and $F \subset E$ is a submanifold. Then, $F$ is called a subbundle of $E$ if there exists an open covering $\left\{U_{i}\right\}$ and transition functions $g_{i j}: U_{i} \cap U_{j} \rightarrow \mathrm{GL}(r, \mathbb{C})$ for $F$ and $h_{i j}: U_{i} \cap U_{j} \rightarrow$ $\mathrm{GL}(n, \mathbb{C})$ for $E$ such that

$$
h_{i j}(x)=\left(\begin{array}{cc}
g_{i j}(x) & * \\
0 & f_{i j}(x)
\end{array}\right) .
$$

The quotient bundle $G=E / F$ is described by transition functions $f_{i j}$.

Now we are ready to define the stability of a vector bundle.

Definition 5.2. A vector bundle, $E$ on a curve, is stable (resp. semi-stable) if every nonzero vector subbundle $F \subset E$ satisfies

$$
\frac{\operatorname{deg} F}{\operatorname{rk} F}<\frac{\operatorname{deg} E}{\operatorname{rk} E} \quad(\text { resp. } \leq) .
$$


(Or equivalently, we can also say that a vector bundle $E$ is stable (resp. semi-stable) if $\frac{\operatorname{deg} G}{\operatorname{rk} G}>\frac{\operatorname{deg} E}{\operatorname{rk} E}($ resp. $\geq$ ) for every non-zero quotient $G$ of $E)$.

From this definition we can see that a vector bundle $E$ of rk 2 is stable (resp. semi-stable) if every line subbundle $F \subset E$ satisfies

$$
\operatorname{deg} F<\frac{1}{2} \operatorname{deg} E \quad(\text { resp. } \leq) .
$$

We call the rational number $\frac{\operatorname{deg} E}{\operatorname{rk} E}$ the slope of $E$. The picture below illustrates the reason for this name.

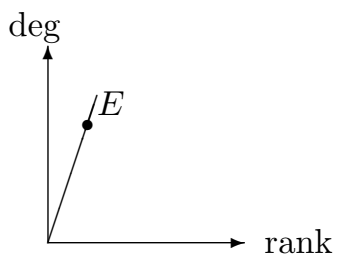

The definition of stability above is often referred to as slope-stability.

Lemma 5.3. Let $E$ be a vector bundle of rank 2. If $\operatorname{deg} E$ is odd, then stability and semi-stability are equivalent.

Proof. Clearly if $E$ is stable, then $E$ is semi-stable. For the other direction, we assume $E$ is semi-stable with degree $n$. Now let $F \subset E$ be a nonzero subbundle of $E$, so $\operatorname{deg} F \leq \frac{n}{2}$. Since $\operatorname{deg} F$ is an integer, $\operatorname{deg} F \neq \frac{n}{2}$ as $n$ is odd. Hence $\operatorname{deg} F<\frac{n}{2}$, i.e. $E$ is stable.

Lemma 5.4. If $E_{1}$ and $E_{2}$ are semi-stable, and $\frac{\operatorname{deg} E_{1}}{\operatorname{rk} E_{1}}>\frac{\operatorname{deg} E_{2}}{\operatorname{rk} E_{2}}$, then $\operatorname{Hom}\left(E_{1}, E_{2}\right)=0$

Proof. Let $f: E_{1} \rightarrow E_{2}$ be a morphism, and let $F \subset E_{2}$ be it's image. Since $E_{2}$ is semi-stable, if $F \neq 0$, then $\frac{\operatorname{deg} F}{\operatorname{rk} F} \leq \frac{\operatorname{deg} E_{2}}{\operatorname{rk} E_{2}}$. But $E_{1}$ is semi-stable and $F$ is a quotient of $E_{1}$, and therefore $\frac{\operatorname{deg} E_{1}}{\operatorname{rk} E_{1}} \leq \frac{\operatorname{deg} F}{\operatorname{rk} F}$, a contradiction unless $F=0$.

5.1. Jordan-Hölder Filtrations. Consider a rational number $\mu$ and let $C(\mu)$ denote the category of semi-stable vector bundles of slope $\mu$. This turns out to be an abelian category (See [8] Chapter 5 for more details). This allows us to define Jordan-Hölder filtrations for each semi-stable bundle: these filtrations are important in order to understand the points of the moduli space of stable vector bundles of rank 2 and degree $d$ (or indeed any fixed rank and degree). 
Definition 5.5. Let $E$ be a semi-stable vector bundle of slope $\mu$. A Jordan-Hölder filtration of $E$ is a filtration of vector subbundles

$$
0 \subset E_{1} \subset E_{2} \subset \cdots \subset E_{k}=E
$$

in $C(\mu)$ such that the quotient $\operatorname{gr}_{i}=E_{i} / E_{i-1}$ is a stable bundle in $C(\mu)$. The integer $k$ is called the length of the filtration and the direct sum $\bigoplus_{i} \operatorname{gr}_{i}$ is called the associated grading.

Let us now see how to get such a filtration. Consider first if $E$ is stable, i.e. $\forall E_{i} \subset E, \mu\left(E_{i}\right)<\mu(E)$. In this case, the filtration is clear. Namely

$$
0 \subset E_{1}=E
$$

Now we consider when $E$ is strictly semi-stable, then there exists subbundles in $C(\mu)$ and one of these, $E_{1}$ must be stable. If not then we can construct an infinite descending sequence of subbundles in $C(\mu)$ in which the rank strictly decreases but this is impossible as the rank of nonzero subbundles of $E$ is bounded below by 1 . So $E / E_{1}$ is also in $C(\mu)$ and we can continue the construction until we obtain our filtration as above.

\subsection{Harder-Narasimhan Filtrations.}

Lemma 5.6. (a) Let $d, d^{\prime}, r, r^{\prime} \in \mathbb{Z}$ with $r, r^{\prime}>0$.

(i) If $\frac{d}{r}>\frac{d^{\prime}}{r^{\prime}}$, then $\frac{d}{r}>\frac{d+d^{\prime}}{r+r^{\prime}}>\frac{d^{\prime}}{r^{\prime}}$.

(ii) If $\frac{d}{r}=\frac{d+d^{\prime}}{r+r^{\prime}}$ or $\frac{d^{\prime}}{r^{\prime}}=\frac{d+d^{\prime}}{r+r^{\prime}}$ then $\frac{d}{r}=\frac{d^{\prime}}{r^{\prime}}$.

(b) Let $0 \rightarrow E^{\prime} \rightarrow E \rightarrow E^{\prime \prime} \rightarrow 0$ be a short exact sequence of nonzero vector bundles on $X$.

(i) If $\lambda \in \mathbb{R}$ such that $\mu\left(E^{\prime}\right) \leq \lambda$ and $\mu\left(E^{\prime \prime}\right) \leq \lambda$, then $\mu(E) \leq \lambda$.

(ii) If $\mu\left(E^{\prime}\right)=\mu(E)$ or $\mu(E)=\mu\left(E^{\prime \prime}\right)$ then $\mu\left(E^{\prime}\right)=\mu\left(E^{\prime \prime}\right)$.

(c) If $0=E_{0} \subset E_{1} \subset E_{2} \subset \cdots \subset E_{n}=E$ is a filtration by subbundles of $E$ such that $\mu\left(E_{i} / E_{i-1}\right) \leq \lambda$ for all $i=1, \ldots, n$ :

(i) then $\mu\left(E_{i}\right) \leq \lambda$ for all $i=1, \ldots, n$. In particular, $\mu(E) \leq \lambda$.

(ii) If, for at least one $i$, we have $\mu\left(E_{i} / E_{i-1}\right)<\lambda$, then $\mu(E)<\lambda$.

Proof. (a) The proof of this is a simple calculation.

(b) Because of the fact that $\operatorname{rk}(E)=\operatorname{rk}\left(E^{\prime}\right)+\operatorname{rk}\left(E^{\prime \prime}\right)$ and $\operatorname{deg}(E)=$ $\operatorname{deg}\left(E^{\prime}\right)+\operatorname{deg}\left(E^{\prime \prime}\right)$, this follows immediately from (a).

(c) This follows from (b) using exact sequences

$$
0 \rightarrow E_{i-1} \rightarrow E_{i} \rightarrow E_{i} / E_{i-1} \rightarrow 0
$$

for all $i=2, \ldots n$. 
Each vector bundle admits a canonical increasing filtration whose successive quotients are semi-stable. This allows us to classify bundles which are not semi-stable in terms of semi-stable bundles.

Proposition 5.7. Let $E$ be a vector bundle on a curve $X$. Then $E$ has an increasing filtration by vector subbundles

$$
0=E_{0} \subset E_{1} \subset E_{2} \subset \cdots \subset E_{k}=E
$$

where the quotient $\operatorname{gr}_{i}=E_{i} / E_{i-1}$ satisfies the following conditions:

(1) the quotient $\mathrm{gr}_{i}$ is semi-stable;

(2) $\mu\left(\mathrm{gr}_{i}\right)>\mu\left(\mathrm{gr}_{i+1}\right)$ for $i=1, \cdots, k-1$.

Proof. ([8] Proposition 5.4.2) If $E$ is already semi-stable then the result is trivial. Assume, therefore that $E$ is not semi-stable. We will prove this by induction on the rank of $E$. If $\operatorname{rk}(E)=1$, then the result is trivial as all line bundles are automatically stable. Now assume $\operatorname{rk}(E) \geq 2$. We know, from Lemma 2.23, that the degree of all subbundles of $E$ is bounded above. On the other hand, subbundles can only have ranks $1,2, \ldots, \operatorname{rk}(E)-1$, hence the slope of the subbundles of $E$ is bounded above. Among all the subbundles of maximal slope, let $E_{1}$ be the one of maximal rank. Then $E_{1}$ is semi-stable because it has maximal slope. Let $E^{\prime}=E / E_{1}$, then we have the following short exact sequence:

$$
0 \rightarrow E_{1} \rightarrow E \rightarrow E^{\prime} \rightarrow 0
$$

where $\operatorname{rk}\left(E^{\prime}\right)<\operatorname{rk}(E)$.

By inductive assumption $E^{\prime}$ has an increasing filtration satisfying the conditions of the proposition, i.e.

$$
0 \subset F_{2} \subset F_{3} \subset \cdots \subset F_{k}=E^{\prime}
$$

with

$$
\mu\left(F_{2}\right)>\mu\left(F_{3} / F_{2}\right)>\cdots>\mu\left(F_{k} / F_{k-1}\right)
$$

and $F_{j} / F_{j-1}$ is semi-stable for $2 \leq j \leq k$. In particular, $F_{2}$ is semistable.

Let $E_{j} \subset E$ be the preimage of $F_{j} \subset E^{\prime}$ under $E \rightarrow E^{\prime}$. This way we obtain commutative diagrams with exact rows:

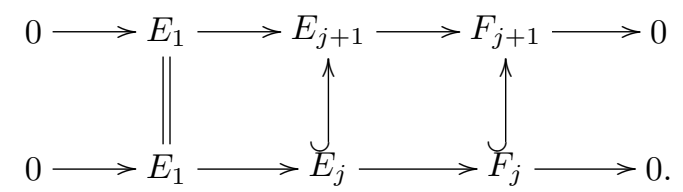


Hence $E_{j+1} / E_{j} \cong F_{j+1} / F_{j}$ are semi-stable.

Now we need to prove $\mu\left(F_{2}\right)<\mu\left(E_{1}\right)$, in order to show condition 2 holds. Since $E_{1}$ has maximal slope, $\mu\left(E_{2}\right) \leq \mu\left(E_{1}\right)$. Moreover, since $E_{1}$ has maximal rank among the subbundles with slope $\mu\left(E_{1}\right), \mu\left(E_{2}\right)<\mu\left(E_{1}\right)$. From the diagram above we know that $\operatorname{deg}\left(F_{2}\right)=\operatorname{deg}\left(E_{2}\right)-\operatorname{deg}\left(E_{1}\right)$ and $\operatorname{rk}\left(F_{2}\right)=\operatorname{rk}\left(E_{2}\right)-\operatorname{rk}\left(E_{1}\right)$. So we know $\mu\left(F_{2}\right)=\frac{\operatorname{deg}\left(E_{2}\right)-\operatorname{deg}\left(E_{1}\right)}{\operatorname{rk}\left(E_{2}\right)-\operatorname{rk}\left(E_{1}\right)}$. We can also write this as $\mu\left(F_{2}\right)=$ $\frac{\operatorname{rk}\left(E_{2}\right) \mu\left(E_{2}\right)-\mathrm{rk}\left(E_{1}\right) \mu\left(E_{1}\right)}{\operatorname{rk}\left(E_{2}\right)-\operatorname{rk}\left(E_{1}\right)}$. Then we have

$$
\frac{\operatorname{rk}\left(E_{2}\right) \mu\left(E_{2}\right)-\operatorname{rk}\left(E_{1}\right) \mu\left(E_{1}\right)}{\operatorname{rk}\left(E_{2}\right)-\operatorname{rk}\left(E_{1}\right)}<\frac{\operatorname{rk}\left(E_{2}\right) \mu\left(E_{1}\right)-\operatorname{rk}\left(E_{1}\right) \mu\left(E_{1}\right)}{\operatorname{rk}\left(E_{2}\right)-\operatorname{rk}\left(E_{1}\right)}
$$

i.e. $\mu\left(F_{2}\right)<\mu\left(E_{1}\right)$. Now since $E_{2} / E_{1}=F_{2}$, we have $\mu\left(E_{1}\right)>$ $\mu\left(E_{2} / E_{1}\right)$. We can the repeat the process until we obtain a quotient $E / E_{k-1}$ which is semi-stable.

Lemma 5.8. If

$$
0=E_{0} \subset E_{1} \subset \cdots \subset E_{n}=E
$$

is a filtration of $E$ satisfying the conditions of Proposition 5.7 above and $E^{\prime} \subset E$ is a nontrivial subbundle of $E$ then $\mu\left(E^{\prime}\right) \leq \mu\left(E_{1}\right)$ and if $\mu\left(E^{\prime}\right)=\mu\left(E_{1}\right)$, then $E^{\prime} \subset E_{1}$.

Proof. We define a filtration of $E^{\prime}$ by $E_{i}^{\prime}:=E^{\prime} \cap E_{i}$ for all $i=$ $1, \ldots, n$. Because $E_{i}^{\prime}=E_{i} \cap E_{i+1}^{\prime}$ we obtain $E_{i+1}^{\prime} / E_{i}^{\prime} \subset E_{i+1} / E_{i}$ for $i=1, \ldots, n-1$. Now since $E_{i+1} / E_{i}$ is semi-stable, we have either $\mu\left(E_{i+1}^{\prime} / E_{i}^{\prime}\right) \leq \mu\left(E_{i+1} / E_{i}\right)$ or $E_{i+1}^{\prime}=E_{i}^{\prime}$. Because $\mu\left(E_{i+1} / E_{i}\right) \leq$ $\mu\left(E_{1}\right)$ for $i=1,2, \ldots, n-1$, we obtain from Lemma 5.6 (c) that $\mu\left(E^{\prime}\right) \leq \mu\left(E_{1}\right)$. Now if $i \geq 1$ and $E_{i+1}^{\prime} \neq E_{i}^{\prime}$ then $\mu\left(E_{i+1}^{\prime} / E_{i}^{\prime}\right) \leq$ $\mu\left(E_{i+1} / E_{i}\right)<\mu\left(E_{1}\right)$. Hence by Lemma 5.6 (c) again, if $\mu\left(E^{\prime}\right)=$ $\mu\left(E_{1}\right)$ we must have $E_{i+1}^{\prime}=E_{i}^{\prime}$ for $i=1,2, \ldots, n-1$, i.e. $E^{\prime} \subset$ $E_{1}$.

Proposition 5.9. This filtration of Proposition 5.7 is unique.

Proof. ([8], Proposition 5.4.2) Assume $\left(E_{i}\right)_{i=1, \ldots, n}$ and $\left(F_{j}\right)_{j=1, \ldots, m}$ are two filtrations of $E$ satisfying the conditions of Proposition 5.7 above. Now using the notation of Lemma 5.8 if we let $E^{\prime}:=F_{1}$ we get $\mu\left(F_{1}\right) \leq \mu\left(E_{1}\right)$. Similarly if we allow $E^{\prime}:=E_{1}$, we get $\mu\left(E_{1}\right) \leq \mu\left(F_{1}\right)$. Clearly then, $\mu\left(F_{1}\right)=\mu\left(E_{1}\right)$.

Lemma 5.8 again implies $E_{1} \subset F_{1}$ and $F_{1} \subset E_{1}$, hence $E_{1}=F_{1}$. Using $E / E_{1}$ and $F / F_{1}$ we can proceed by induction as in the proof of Proposition 5.7 to conclude that the filtration is unique. 
The filtration of Proposition 5.7 is called the Harder-Narasimhan filtration of $E$.

\section{Conclusion}

In conclusion, it is fair to say that the theory of vector bundles is vast and indeed very interesting. We have seen how vector bundles on $\mathbb{P}^{1}$ are not very complex, in the sense that they can be written as a direct sum of line bundles. We have also seen a classification for indecomposable rank 2 vector bundles on elliptic curves.

One could also go on to study higher rank vector bundles on elliptic curves or on curves of a higher genus, or even on higher dimensional complex manifolds. These are all very interesting in their own right.

In Section 5, we studied slope stability for vector bundles on curves. As was mentioned, stable bundles are required when constructing moduli spaces of vector bundles. For the reader interested in stability, from here you could go on to study Bridgeland stability conditions ([2] and [3]) and the space of all stability conditions on a particular complex manifold (e.g., an elliptic curve).

\section{REFERENCES}

[1] M. F. Atiyah: Vector bundles over an elliptic curve, Proc. London Math. Soc. (3) 7 (1957), 414-452.

[2] T. Bridgeland: Stability Conditions on Triangulated Categories, preprint arXiv:math.AG/0212237.

[3] T. Bridgeland: Spaces of Stability Conditions, arXiv:math.AG/0611510.

[4] A. Gathmann: Algebraic Geometry, Notes for a class taught at the University of Kaiserslauten (2002/2003) available at http://www.mathematik.uni$\mathrm{kl}$.de/ gathmann/class/alggeom-2002/main.pdf

[5] R. Hartshorne: Algebraic Geometry, Graduate Texts in Mathematics, Springer, 1977.

[6] D. Huybrechts, M. Lehn: The geometry of moduli spaces of sheaves Aspects of Mathematics, E31. Friedr. Vieweg \& Sohn, Braunschweig, 1997.

[7] M. Khalid: Group law on the cubic curve, this issue.

[8] J. Le Potier: Lectures on Vector Bundles Cambridge studies in advanced mathematics, Cambridge University Press, 1997.

[9] S. Mukai: An Introduction to Invariants and Moduli, Cambridge studies in advanced mathematics, Cambridge University Press, 2003.

[10] C. A. Weibel: An introduction to homological algebra, Cambridge studies in advanced mathematics, Cambridge University Press, 1994. 
Ciara Daly,

Mary Immaculate College,

South Circular Road,

Limerick, Ireland

ciara.daly@mic.ul.ie

Received in final form on 23 August 2007. 